\title{
EDITORIAL
}

\section{Genomic sciences for developmentalists: The current state of affairs}

\author{
ELENA L. GRIGORENKO ${ }^{a-c}$ AND DANTE CICCHETTI ${ }^{d, e}$ \\ ${ }^{a}$ Yale University; ${ }^{b}$ Columbia University; ${ }^{c}$ Moscow State University; ${ }^{d}$ Institute of Child Development, University of Minnesota; and \\ ${ }^{e}$ Mt. Hope Family Center, University of Rochester
}

Our work on this Special Issue has been an extremely interesting experience. We had a chance to review and comment on the work of a number of leading scholars in the field of genetic and genomic sciences and had the opportunity to reflect on the status of the field, now approaching the 10th anniversary of the finalization of the first sequence of the human genome in 2003 (Human Genome Program, 2008). These 10 years have been fundamentally important, even revolutionary, for the field of genomics and genetics. They have been no less important for fields of science that use genomic and genetic knowledge to advance their cause: psychiatry and psychology in general, and developmental psychopathology in particular.

Despite concerns that the early expectations and promises of the impact of genetic and genomic sciences on psychology and related sciences (e.g., Plomin \& Rutter, 1998) have been overly optimistic, we subscribe to the statement by Collins (2010). Reflecting on the technological developments triggered by the Human Genome Project, Collins stated: "The consequences of a radical new technology are almost always overestimated in the short term and underestimated in the long term"' (p. 268). It is in the spirit of this deterministic expectation that we have put this collection of articles together.

The Special Issue is organized along a number of dimensions, cohesively sampling from the broad landscape of the state of affairs in the field. Its five major contributions can be summarized along the following lines. First, these articles present a vision for the continued advancement of the relationships between the genetic and genomic sciences and psy-

Our work on this Special Issue was supported by the National Institutes of Mental Health Grant MH082592 (to E.L.G.) and Grant MH083979 (to D.C.), National Institute on Drug Abuse Gramt DA017741 (to D.C.), and the Spunk Fund (to D.C.).

Address correspondence and reprint requests to: Elena L. Grigorenko, Child Study Center, Yale University, 230 South Frontage Road, New Haven, CT 06519; E-mail: elena.grigorenko@yale.edu; or Dante Cicchetti, Institute of Child Development, University of Minnesota, 51 East River Road, Minneapolis, MN 55455; E-mail: cicchett@umn.edu. chiatry and psychology in general and developmental psychopathology in particular. Specifically, although all of the contributions to this Special Issue contain explicit discussions of numerous difficult issues faced by researchers working at the interface of the genetic and genomic sciences and developmental psychopathology, all of the articles are marked by optimism and content. Although the field is not where it was expected to be a decade ago, the past decade has been a source of tremendous discoveries, the relevance of which for understanding the pathways of typical and atypical human development is impossible to underestimate.

Second, this collection presents a blend of reviews and empirical studies from the field. Thus, the articles delineate the frontiers of current research and exemplify these frontiers with empirical illustrations.

Third, the articles describe and typify the major technological advancements that have characterized the last decade. These are genome-wide association studies, whole-genome sequencing for DNA and RNA, microarray technologies, statistical developments pertaining to the analyses of gene-environment interplay, and technological applications for the generation and analyses of the induced polypotent stem cells.

Fourth, these articles sample from numerous rapidly developing fields of study, bringing ideas, approaches, and methods from investigations of the genome, epigenome, and proteome to studies of typical and atypical development.

Fifth, and perhaps of the most importance, this collection is true to the vision of the mission of the Journal that hosts it: all articles advance our knowledge of developmental psychopathology (Cicchetti, 1984; Cicchetti \& Cohen, 1995; Rutter \& Sroufe, 2000; Sroufe \& Rutter, 1984) by presenting the current state of the field and outlining its future directions or by providing new data on typical and atypical development.

The Special Issue opens with a contribution by Wendy Johnson, who shares her views and analyses of the role of evolutionary and developmental biology in the current landscape of the field. The article starts with a reminder that the first fundamental discoveries of genetics made by Gregor 
Johann Mendel were possible and limited to completely penetrant genes. However, what has followed in the subsequent century and a half of the development of genetics and now genomics has verified that most genes, especially those in humans, are not completely penetrant. Thus, assuming that it is those incompletely penetrant genes that are most important for personality and psychopathology, it is argued that the field needs a perspective shift in interpreting genetic influences on psychopathology and that such a shift can be substantiated with ideas and data that have been accumulated in the fields of evolutionary and developmental biology. This argument is delivered sequentially and logically. Its presentation starts with a brief summary of Mendel's discovery and its reinterpretation, application to quantitative traits, and enhancement by Sir Ronald Fisher. It then proceeds through a brief discussion of the role of quantitative genetic studies (i.e., twin, adoption, and various other types of blood relatives); makes reference to the work of Conrad Waddington and his vision of the epigenetic landscape, in which he sees the possibility of developing a conceptual model of the interactions between genes and their surroundings that produce a phenotype; and provides a detailed examination of the work of Ivan Schmalhausen. The review reiterates the essentials of what is known as Schmalhausen's law and the attendant principles, extends them, and discusses their applicability in an extended form to studies of human psychopathology in its two perspectives: psychopathology as manifested in the individual and psychopathology as manifested in the population.

The contribution by Lauren M. McGrath, Sydney Weill, Elise B. Robinson, Rebecca MacRae, and Jordan W. Smoller provides an illustration of the profile of research that exemplifies Johnson's claim of the need for a conceptual shift in the field of psychiatric genetics. This article reviews the rather constrained progress in the field in the identification of the genetic bases of anxiety disorders. One of the clear limits of such progress is the phenotypic complexity of anxiety, which is associated with incomplete or variable penetrance and with high rates of comorbidity with other disorders. It is the need for fine-grained phenotypic work that should occur in conjunction with progress in molecular explorations that is central to the discussion in this contribution. The main thrust of this article is its description of how a developmental perspective can help inform, enhance, and define anxiety phenotypes for genetic research. The authors support the importance of such a description using psychiatric and epidemiological data, focusing on the early onset and chronic course of anxiety disorders, and indicators of shared and specific genetic risk factors within and across time and specific symptoms. The authors claim that the lack of progress in the genetics of anxiety is related to difficulties in the definition of anxiety phenotypes and the reduction of etiological and phenotypic heterogeneity. They subsequently propose the usage of three strategies that they refer to as developmentally sensitive phenotypes: an examination of early temperamental risk factors, the use of latent factors to model underlying anxiety liability, and the use of developmental trajectories as pheno- types. The authors argue that the breakthrough in anxiety research should come from a shift from the conceptualization and execution of single-disorder, case-control studies to developmentally oriented multivariate views of anxiety, in which power is maximized by large samples and by virtue of executing multiple measurements at multiple time points.

The contribution by Scott I. Vrieze, William G. Iacono, and Matt McGue provides a comprehensive outline of research approaches to studying first-order (main) and second-order (interactions and correlations) effects of genes and environments on the emergence of atypical developmental pathways and the manifestation of the corresponding disordered phenotypes. The article provides a historical context for genome-wide and candidate-gene studies and discusses the differences and similarities between these designs with regard to their benefits, limitations, and expected payoffs. Then, the authors cover a number of important issues that the authors feel will determine the ultimate outcome of efforts in the field. The first concerns the presence of an adequate psychological theory that could possibly guide the conceptualization of the phenotype and the selection of the study design. The second addresses the measurement issues pertaining to the phenotype as informed by modern psychometrics and the importance for genetic and genomic research to work with phenotypes that are as error free as possible. The third suggests that the selection of a design should be driven by the overall aims of the work and be in concert with the contextualizing psychological theory and the assessment of the target phenotype. Finally, the article focuses on the current challenges presented by today's genetic data: its massive quantities and software challenges, which affect data manipulation and data analyses. The article concludes that the field's improved understanding of the structure and function of the genome should inevitably generate new tools to test specific a priori hypotheses derived from psychological theories; the authors also argue that such hypothesis testing might not be much different from what happens with current candidate-gene hypothesis testing but will be characterized by more replicable findings, less confusion, and a greater feeling of progress. The candidate-gene approach, the inferential machinery of which is predicted to remain vital for studies of developmental psychopathology, is epitomized in a number of contributions to this Special Issue.

The contribution by Megan R. Gunnar, Jennifer A. Wenner, Kathleen M. Thomas, Charles E. Glatt, Morgan C. McKenna, and Andrew G. Clark exemplifies one of the most common realizations of this approach in psychology today, focusing on a single functional genetic variant, the properties of which are known and the presence of which in the literature can be interpreted in a set of specific inferential hypotheses in a well-characterized behavioral sample from a distinct subpopulation. These researchers provide data from a diverse sample of youth adopted internationally into the United States from 25 countries where they resided in orphanages. At the center of their study is one of the variants in the brain-derived neurotrophic factor gene, encoding a secreted protein that is a mem- 
ber of the neurotrophin family of growth factors, the primary role of which is to support the survival of existing neurons and contribute to the molecular environment that conditions the growth and differentiation of new neurons and the establishment of synapses. The brain-derived neurotrophic factor gene contains a functional variant, Val66Met (rs6265), a single nucleotide polymorphism (SNP) in the gene, in which adenine and guanine alleles are both observed in the general population; this SNP results in a variation between valine and methionine at codon 66 . This polymorphism has been studied in the field extensively, although the resulting patterns of findings are contradictory (Mandelman \& Grigorenko, 2012). The behavioral targets of this investigation were quantifiers of attentional problems estimated through parent reports. The genotype (along with gender and country of origin) differentiated the association between the duration of institutional care and indicators of attentional problems. The authors state that the relationships are curvilinear: the number of attention problem symptoms depends on the genotype of the carrier and the time of adoption.

Similarly, Triin Kurrikoff, Klaus-Peter Lesch, Evelyn Kiive, Kenn Konstabel, Sabine Herterich, Toomas Veidebaum, Andreas Reif, and Jaanus Harro focus on a range of behavioral phenotypes associated with the trait of impulsivity and its related psychopathology while considering genetic variation in the encoding enzyme nitric oxide synthase 1 (NOS1) neuronal gene. This gene has a polymorphism in the promoter region of the alternative first exon 1f, referred to as NOS1 ex1f-variable number tandem repeat (VNTR). The gene has multiple isoforms with different first exons (up to 12 potential exons); exons $1 \mathrm{c}$ and $1 \mathrm{f}$ are reported to be transcribed most frequently in the brain. This VNTR is highly polymorphic and is often dichotomized in two alternative clusters referred to as long and short variants for simplicity. The long variant compared to the short variant results in magnified reporter activity. This VNTR has been studied by these investigators, and its associations have been established with impulsivity and related psychopathology in different populations; it has been reported that the carriers of the NOS1 short/short genotype appear to demonstrate more impulsive behaviors and be at higher risk for psychiatric disorders. As stated in this article, this association is not straightforward and is complicated by the gender factor and specific environmental conditions. Correspondingly, this article provides an illustration of how this complexity can be disentangled at least partially within the framework of a longitudinal, population-based sample of adolescents. Specifically, behavioral data collected on indicators of personality, impulsivity, and anxiety at ages 15 and 18 and on indicators of depression assessed only once were modeled against self-reported data of stressful life events at age 18, demographic data, and the genotyping data. The results reaffirm the presence of an association between a specific variant of the NOS1 ex 1f-VNTR previously reported in clinical populations with neuroticism, extraversion, and anxious and depressive traits in the general population. Moreover, this association is differentiated by indicators of gender and stressful life events.

The article by Melissa N. Dackis, Fred A. Rogosch, Assaf Oshri, and Dante Cicchetti provides another example of the candidate-gene approach. The idea behind this work was to investigate the connection between the concept of limbic irritability, which is thought to be at least partially an outcome of increased excitatory neurotransmission following maltreatment and depressive and dissociative behavioral symptomatology. In other words, the authors hypothesized that childhood maltreatment could possibly lead to the manifestation of internalizing psychopathology and that this connection is indirect through limbic irritability. They assumed that limbic system dysfunction was at least partly controlled genetically; thus, they expected the indirect connection between maltreatment and internalizing to be moderated by genetic factors. These factors are sampled in the article through the FK506 binding protein 5 (FKBP5) gene, encoding a protein that is a member of the immunophilin protein family. Proteins in this family are known to be involved in immunoregulation and basic cellular processes involving protein folding and trafficking. The researchers collected DNA from a sample of high-risk, low-income, inner-city women, who also selfreported on child maltreatment, limbic irritability, and symptoms of depression and dissociation. What is especially important about this manuscript is that the FKBP5 gene was sampled through a number of SNPs rather than through a single marker known to be functional or not functional. This approach underlines what has become rather apparent within the last decade or so of genetic research: any gene is a dynamic and complex system that cannot be accurately captured by a single polymorphism and needs to be represented using a multivariate approach, because the function of any variant can be affected by its surroundings (Pritchard \& Przeworski, 2001). The results of this investigation demonstrate that the FKBP5 gene variation, more specifically, a fourSNP haplotype within the gene, moderates the indirect effect of maltreatment via limbic irritability on depression and dissociation.

Sara R. Jaffee and S. Thomas Price continue the discussion of the importance of considering the potential for reverse causation and confounding in any type of research that connects behavior and genes through the whole-genome or candidate-gene methodologies. This potential is associated with two observations. First, most environmental measures are heritable; their impacts on the vulnerability for psychopathology can be confounded by the genetic profile of the individual. Second, genetic risk profiles can be mediated by their effect on environmental risk factors. These two considerations have sparked a new direction of research known as genotypeenvironment correlation. This article provides definitions of this phenomenon and its subtypes (passive, evocative, and active or selective), compares it with the phenomenon of Gene $\times$ Environment $(\mathrm{G} \times \mathrm{E})$ interaction, and reviews the evidence of the importance of genotype-environment correlation obtained from quantitative genetic studies. The article fo- 
cuses more centrally on the evidence of the importance of genotype-environment correlation drawn from the moleculargenetic literature. Specifically, the featured examples cover the correlation between maternal and child dopamine genotype and parental behavior and between maternal oxytocin genotype and maternal behavior. The article concludes with a discussion of the importance of accounting for the influence of exposure to environmental risk factors (i.e., genotypeenvironment correlation) in any attempt to advance genetic and environment epidemiology or to trigger advances in each other. The corresponding causal links are complex and reciprocal: genes exist in environments and in these environments they shape our behaviors and cognitions, which then shape our environments. These interdependencies should be addressed with great care in the developmental sciences generally and particularly developmental psychopathology.

The theme of genotype-environment correlation is further developed in the contribution by Cathi B. Propper, Michael J. Shanahan, Rosemary Russo, and Roger Mills-Koonce, who argue that genotype-parenting evocative correlation (i.e., the sensitivity of parenting in response to the child's dopamine receptors D2 and D4 genotypes) in the first 3 years of life is associated with subsequent psychosocial adjustment and then academic performance in primary school, as evaluated by teachers and by maximum performance tests. This assumption was based both on the literature and on the authors' earlier work. Specifically, using multiple waves of data from the Durham Child Health and Development Study, the researchers demonstrated that gender-differentiated risk alleles from dopamine receptor genes (dopamine receptor D4 genotype for girls and dopamine receptor D2 genotype for boys) are associated with less sensitive parenting. Specifically, the authors claim that parenting mediates the connection between dopamine receptor D4 genotype and all academic outcomes (for girls). According to the model, parenting also influences girls' withdrawn behavior in classroom, which impacts teachers' perception and assessment of academic performance. For boys, parenting appears to be associated with emotional regulation, which impacts teachers' perception and assessment of academic performance. Acknowledging the preliminary nature of their findings, the researchers argue that their model might shed some light on the field's understanding of how evocative correlation in the home might predict facets of psychosocial functioning, which might then impact true academic performance and teachers' appraisals of it. Thus, these findings might provide an initial step toward understanding the role of genetic factors in family functioning and the subsequent causal linkages between the child as an organizer of parenting behavior early in life and the subsequent impact of this child-dependent parenting on academic achievement.

The article by Gordon Harold, Kit Elam, Gemma Lewis, Frances Rice, and Anita Thapar provides yet another example of studies of genotype-environment correlation, but it discusses the role of passive genotype-environment correlation using antisocial behavior as a target of their investigation. Antisocial behavior is coupled with two major risk factors: the intergenerational transmission of antisocial behavior (i.e., higher levels of antisocial behavior in parents serve as risk factors for higher levels of antisocial behavior in children) and family socialization into antisocial behavior (i.e., interparental conflict and negative parenting practices elevate the risk for externalizing behaviors). Given this context, the researchers examined the role of parent to child hostility as a mediating mechanism underlying links between interparental conflict (i.e., an indicator of family socialization), parent antisocial behavior (i.e., an indicator of intergenerational transmission) and child antisocial behavior in the context of a design, including genetically related and genetically unrelated parent-child pairs. This genetically sensitive design was possible through the recruitment of families that utilized in vitro fertilization for their reproductive purposes. The results indicated that, for genetically related mothers, interparental conflict and maternal antisocial behavior indirectly influenced child antisocial behavior through mother to child hostility. For genetically unrelated mothers, effects were apparent only for maternal antisocial behavior on child antisocial behavior through mother to child hostility. Finally, for genetically related and genetically unrelated fathers and children, interparental conflict and paternal antisocial behavior influenced child antisocial behavior through father to child hostility. In short, passive genotype-environment correlation can serve as a possible confounding influence in understanding the specifics of the intergenerational transmission of antisocial behavior and family socialization into violence.

Julia Kim-Cohen and Rebecca Turkewitz further contribute to the discussion of the juxtaposition of genes and environments in human development, but their focus is on $G \times$ $\mathrm{E}$ interaction. Although providing an overview of the state of affairs in the field, they comment on the lack of attention of $\mathrm{G} \times \mathrm{E}$ interaction studies to the concept of resilience, one of the central concepts in the field of developmental psychopathology. The article offers an up-to-date summary of the dynamic field of studies of resilience, starting with its malleable definition, describing its ever-developing models, and illustrating it with examples of measured $G \times E$ interaction in studies of maltreatment. The authors bring up a number of issues pertaining to the importance of adjusting the diagnostic categories used in psychopathology to reflect on the importance of environmental risk (e-risk), understanding the variability in environmental and genetic risk factors as it pertains to the concept of resilience, utilizing the developmental perspective in research on resilience and $\mathrm{G} \times \mathrm{E}$ interaction, and understanding the mechanisms and coverage of resilience to better effect public health benefits and improved mental health.

The candidate gene approach is further exemplified and taken to a different level of abstraction in the article by Colin G. DeYoung and Rachel Clark. In an attempt to resolve the tension between quantitative genetics findings that substantial heritability of nearly all psychological traits and molecular genetics studies have failed to deliver on the understanding of the specific molecular textures of these high heritability estimates (i.e., the tension that is referred to in the literature as 
the missing heritability problem), the authors focus their attention of the concept of Gene $\times$ Trait $(\mathrm{G} \times \mathrm{T})$ interaction. The $\mathrm{G} \times \mathrm{T}$ interaction is introduced in the context of predicting the level of Trait Y so that there is an interaction term in the predictive equation between a genetic variant and Trait $X$. The main premise underlying the argument for the $G \times$ $\mathrm{T}$ interaction concept is that genes and environments are distal, whereas the brain is a proximal operator of behavior. The impact of any single genetic variant and of any single environmental factor depends on the configuration and status of the brain that translates its impact into behavior. Although measures of the brain (i.e., its time- and place-specific configuration and status) are desirable, they are often unattainable. However, a psychological trait (Trait X) can be viewed as a characteristic pattern of psychological function (i.e., a proxy for brain function), which reflects the impacts of all relevant genetic variants and all relevant environments and cumulatively captures a broad organismic context in which the effect of any single variant unfolds. This article is a review in which the concept of $\mathrm{G} \times \mathrm{T}$ interaction and its examples are discussed through the analyses of different bodies of work. For example, while developing this concept, the authors reviewed the literature on neighboring terms such as $\mathrm{G} \times \mathrm{E}$ and Gene $\times$ Gene interaction. The authors complete their article by prescribing to $\mathrm{G} \times \mathrm{T}$ interaction's great promise in the field's endeavors to understand the interactions between specific genetic variants and the organism as a whole; these interactions, it is argued, are what produces phenotypic consequences.

Another possibility for the resolution of the missing heritability problem, in addition to searching for various secondrather than first-order (i.e., main) effects that can explain high heritability estimates in the absence (or mere absence) of main effects, is to redefine main effects. This possibility is related to the realization that the human genome, which has many such variants or SNPs (predominantly considered to be the source of main effects in genome-wide association studies), carries structural variants of different types; although smaller as a category than that of SNPs, each of these collectively contributes to a large repository of variation. These types of structural variation in the genome are reviewed and exemplified for this Special Issue by Michael Gill and James J. H. Rucker, and Peter McGuffin in their respective articles. Collectively, these two contributions review major features of the technologies that have resulted in the description and quantification of these variants: comparative genome hybridization (CGH) arrays, SNP chips, and DNA sequencing (DNA-Seq). They then present new numerous examples of associations between type and number of a wide range of structural variants and different developmental disorders. The articles differentiate common and rare, recurrent and de novo, and various types (e.g., insertions, deletions, inversions, duplications) of variants and provide a comprehensive overview of today's literature on specific developmental disorders. They discuss the developmental disorders in which the role of such variants was shown to be causative (i.e., intellectual disabilities and nonspecific learning disabilities as exemplified by specific genomic disorders such as Wolf-Hirschhorn, Williams, Langer-Giedion, Prader-Willi and Angelman, Rubinstein-Taybi, Smith-Magenis and Potocki-Lupski, Miller-Dieker, DiGeorge, and other syndromes) and in which it was shown to be important but only probabilistic (i.e., autism spectrum disorders, schizophrenia, attention-deficit/hyperactivity disorder). There is also a summary of the putative mechanisms of the emergence of these variants. Moreover, these articles provide discussions for the potential of using the work on structural variants for diagnostic and treatment purposes in practice and for the concept of shared neurobiology for theories of developmental disorders. Yet, although rich in content and celebratory in attitude, applauding the progress in the field of neuropsychiatric genetics, the articles also deliver a cautionary note. This is driven by a crystal clear realization of the gap between genetic variation and the variation in observed human behavior. At this point, this gap is rather wide and is substantiated by a number of circumstances, including the following: the impossibility of translating a registered association into a causal link because experimentation in humans is unattainable; the large amount of instability of the registered associations and their lack of replicability across samples, designs, and laboratories; and, as pointed out by Wendy Johnson in the first article, a strong likelihood of the incomplete penetrance for any structural variant which is common (i.e., $>1 \%$ ) at the population level. Although the explanatory power of structural variants appears to be greater than that of SNPs at present, the jury is still out. Having presented a comprehensive account of the field, the authors refer to the polygenetic, complex model of neuropsychiatric disorders explicitly proposed almost a half a century ago by Irving Gottesman and James Shields (1972), rooted in even earlier work by Falconer (1965), as the leading model of the field. As a result, their conclusion is that much work remains to be done. However, the research on structural variants represent a real advance in psychiatric genetics and developmental psychopathology, the most promising that the field has had so far.

Whatever structural variants are considered, whether they are SNPs, VNTRs, or any other type, the question of the selection of the phenotype for genetic/genomic analyses remains critical regardless. In this light, Adam Naples, Len Katz, and Elena Grigorenko bring up the important issue of how we might understand the genetic architecture of complex traits (e.g., developmental disorders) by decomposing them into what are thought to be traits of hierarchically lower levels more proximal to the origin of the problem (i.e., to the functional properties of the brain and the genome). Such a decomposition, first introduced by Bernard John and Kenneth R. Lewis in their work with the genetics of the grasshopper, was presented to the field of neuropsychiatric genetics by Irving Gottesman and Todd Gould (2003) and has received a welcome reception. This has resulted in the generation of many studies where the facets of a complex holistic phenotype (e.g., schizophrenia) are viewed as numerous lower level 
phenotypes (e.g., eye saccades or specific characteristics of evoked potentials), and these endophenotypes are subsequently associated with different sources of genetic and genomic variation. Specifically, based on the literature, the researchers evaluated the propensity of lexical decision to serve as an endophenotype for reading comprehension. As deficits in reading comprehension are common to many developmental disorders, it is possible that there might be an endophenotype that is shared between these disorders and can lead investigators to understanding their vulnerability at the endophenotypic levels; this might explain correlations between disorders that are often comorbid, but are currently perceived and treated as distinct clinical entities. The authors' analyses reveal that there is little or no overlap between the genes contributing to lexical decision and reading comprehension and that the genetic mechanism behind lexical decision derived from this analysis appears to be more complex than that for reading comprehension. The researchers hypothesize putative explanations for this finding and propose complementary courses of action that may verify these hypotheses.

A separate set of inquiries into the epigenome opens with a contribution by Catherine Monk, Julie Spicer, and Frances Champagne. This article sets the stage for a discussion of epigenetic mechanisms, especially their role in mirroring early developmental adverse affects (particularly maternal distress) for adaptive and maladaptive life span outcomes. They review evidence illustrating the association among maternal prenatal distress, its association with fetal and infant developmental trajectories, and the potential role of epigenetic mechanisms in mediating this association. Their analyses of the literature start with a summary on prenatal exposure; they then present data on the placenta's high susceptibility to maternal distress and its subsequent vulnerability to epigenetic dysregulation, finishing with a discussion of the role of postnatal maternal care and related epigenetic mechanisms. The review covers a large variety of topics and types of studies, including epidemiological, prospective event-based (e.g., follow-up studies of natural disasters), longitudinal-observational, and mechanistic, that focus on fetuses and infants. Their description illuminates the context of epigenetic mechanisms as reflections of the putative biological machinery underlying the connection between early developmental adversity and subsequent maladaptive developmental outcomes. Epigenetic data available for humans are still scarce, so specific illustrations are provided with examples from the literature on animal models. The review is enriched with a discussion of critical methodological issues and empirical questions that need to be addressed prior to soliciting large systematic amounts of data that might enhance our understanding of the mechanisms of prenatal development. Among these issues are debates pertaining to the specificity versus generalizability of epigenetic patterning obtained from human peripheral tissue samples and the role of animal models and their advantages and disadvantages for understanding epigenetic mechanisms in humans. Overall, this discussion serves as a perfect introduction to the following four articles.
Valerie S. Knopik, Matthew A. Maccani, Sarah Francazio, and John E. McGeary exemplify in detail one of the themes of the Monk and colleagues' review, which is the impact of adverse intrauterine conditions and exposure on subsequent development, by scrutinizing in detail the literature on maternal cigarette smoking. This is viewed as a common hazardous risk factor associated with decreased birth weight, suboptimal developmental outcomes, and elevated risk for psychopathology in later life. The accumulation of recent literature indicates that maternal smoke appears to be one of the triggers of specific epigenetic events in the fetus; it has been associated with anomalous DNA methylation and altered expression of microRNA. The article dealves into this literature and presents a summary of the evidence connecting maternal cigarette smoking during pregnancy with epigenetic alterations and whether and how these alterations impact prenatal and postnatal growth, development, and health. The authors summarize the corresponding literature and cover such important concepts as a critical window, the epidemiology and etiology of cigarette smoking by pregnant women, particularly relevant elements of the theory of fetal programming and the potential of epigenetic mechanisms as molecular mechanisms underlying this programming, and the role of maternal smoking as a factor causing aberrations in DNA methylation and microRNA expression. The article concludes with the importance of studying the molecular impact of maternal smoking during pregnancy as a model of early adverse exposures and their consequences for development and adult life, emphasizing the urgency of combining different molecular technologies to better understand the sources of genetic, epigenetic, and proteomic variation in seeking as well as processing exposure to teratogenic agents such as nicotine. According to the authors, the promise of advanced understanding is in the utilization of multiple technologies, longitudinal methods of analyses, and complex modeling techniques.

The article by Stephen G. Lindell, Qiaoping Yuan, Zhifeng Zhou, David Goldman, Robert C. Thompson, Juan F. Lopez, Stephen J. Suomi, J. Dee Higley, and Christina $\mathrm{S}$. Barr further explores the existence of the genome in a particular environment (or environments). The authors present their work featuring molecular characteristics of hippocampi dissected from male rhesus macaques: half were reared for the first 6 months by peers and half were reared by mothers. They argue that the former group of animals experienced a large amount of stress, whereas the latter was brought up in more typical, more protective (i.e., less stressful) conditions. In their work, the researchers have combined two technologies. First, they genotyped the monkeys for a common polymorphism in the promoter region of the serotonin transporter gene (also known as SLC6A4). This polymorphism has been intensively studied in humans and other animals, including rhesus monkeys (Caspi et al., 2010). Second, they manipulated the hippocampus tissue to investigate particularities of the regional serotonin transporter gene epigenetic modifications, for example, the methylation profile of the studied 
gene, specifically histone $\mathrm{H} 3$ trimethylated at lysine 4 (H3K4me3) binding. These analyses were performed using the sequencing of chromatin immunoprecipitated DNA. Third, in a set of interaction analyses, they studied whether levels of $H 3 K 4 m e 3$ binding are influenced by early rearing history (i.e., dependent on stress) and age. Specifically, their findings revealed that the studied epigenetic marker was lower in peer-reared compared to mother-reared monkeys and in monkeys of postadolecent versus preadolescent age. It is interesting that, although there were no effects of the serotonin transported genotype on the levels of $H 3 \mathrm{~K} 4 \mathrm{me} 3$ binding ubiquitously, there were genotype-specific epigenetic modifications in the gene itself. Moreover, there were age, genotype, and rearing history interactive effects on the levels of the serotonin metabolite 5-hydroxyindoleacetic acid in the cerebrospinal fluid (e.g., a measure of central serotonin reuptake) in a larger sample of rhesus monkeys. Thus, these findings provide an example of a candidate gene-based study, where an investigation of the structural variation within the gene is coupled with an investigation of epigenetic variation across the gene that is assumed to be specific to particular developmental stages and environmental contexts.

Becky C. Carlyle, Alvaro Duque, Robert R. Kitchen, Kelly Bordner, Daniel Coman, Eliza Doolittle, Xenophonios Papademetris, Fahmeed Hyder, Jane R. Taylor, and Arthur A. Simen present a set of results from their work with a rodent maternal separation with early weaning (MSEW) model. The model was designed in an attempt to provide comprehensive molecular profiling of outcomes of early life neglect. This particular contribution, capitalizing on previous results, presents data on the sequencing of chromatin immunoprecipitated DNA, diffusion tensor magnetic resonance imaging, and new behavioral analyses. These new results are embedded in the findings generated by the MSEW model earlier (i.e., the presentation of behavior, the anatomy, and protein expression profiles that are generally highly reflective of that observed in human survivors of early life maltreatment). This article provides new empirical data on the presence of behavioral attention deficits; striking changes in factional anisotropy in rostral areas of the brain, including those implicated in executive function and inhibitory control; a large-scale negative bias in protein expression and the downregulation of myelin-associated genes; and changes in the whole-genome patterns of $H 3 K 4 m e 3$ binding, that is, the downregulation of a modification, especially in promoter regions, that results in an altered epigenetic signature. Thus, the availability of the murine MSEW model of early life neglect creates a possibility for the comprehensive genomic and proteomic characterization of, and genetic and pharmacological manipulations with, animals that have undergone early life neglect. Although these and previous findings have provided interesting and valuable data, the authors acknowledge that more research is needed to determine the face validity, predictive power, efficacy and effectiveness of the paradigm in modeling human disorders that are thought to be connected to early life stress.
The importance of understanding molecular mechanisms associated with early adverse experiences is further exemplified in the article by Johanna Bick, Oksana Yu. Naumova, Scott Hunter, Baptiste Barbot, Maria Lee, Suniya S. Luthar, Adam Raefski, and Elena L. Grigorenko. This contribution brings the discussion back to the level of humans (i.e., where it was started in the contribution by Monk and colleagues). It is structured as a literature review with two empirical illustrations, and it revolves around the role of epigenetic alterations, in response to neglect and abuse, in two key biological systems: the hypothalamus-pituitary-adrenal axis and the immune system. It is important that this article further exemplifies the two leading methodological approaches to understanding the role of epigenetic regulation: the whole-genome approach and the candidate-gene approach. Using specimens donated by young adults and their mothers with and without the involvement of the Department of Children and Families, whose involvement had resulted in early developmental separation and child placement outside of the family, the researchers illustrate how explorations of genome-wide and candidate-gene DNA methylation might inform hypotheses and guide future research efforts at the cross-roads of behavioral and molecular research. Specifically, using a genomewide approach, the authors illustrate how the experience of entering foster care in childhood is associated with the methylation profiles of an array of genes functionally implicated in immune system activity. By exploring methylation levels of select candidate genes, the authors concurrently show how histories of maternal warmth or rejection are associated with methylation levels of specific loci on genes involved in the hypothalamus-pituitary-adrenal axis and immune system activity. Although preliminary, these findings provide further evidence that the characteristics of social environments are traceable in the characteristics of epigenetic patterning and that those traces are detectable at the levels of the whole genome and specific candidate genes.

The last two articles presented in this Special Issue address the utilization of methods and technologies that are novel for the field of psychiatry and psychology in general and developmental psychopathology in particular.

Oksana Yu. Naumova, Dean Palejev, Natalia V. Vlasova, Maria Lee, Sergei Yu. Rychokov, Olga N. Babich, Flora M. Vaccarino, and Elena L. Grigorenko make the case for more active utilization of the study of gene expression (or the transcriptome) in the field. This article provides a brief overview of the relevant literature and focuses on an empirical comparative study of gene expression across different developmental periods (i.e., capturing age-related differences in expression) and different brain areas (i.e., capturing regionspecific differences in expression). Similar to what was done in this Special Issue in the studies of structural genetic variants and epigenetic studies, the authors provide a broad description of appropriate technologies (DNA-Microarray and RNA-Seq, also known as whole transcriptome shotgun sequencing of RNA) and examples of the utilization of these technologies in the literature. This review is understandably 
focused on studies of gene expression in the human brain, which have contributed evidence attesting to the structure-, function-, and age-related variability of the transcriptome in the human brain. The discussion illustrates (when possible) and interprets (when impossible) the presented literature with regard to the principles of developmental psychopathology. The review provides the context for an empirical illustration of the related concepts and technologies: an RNA-Seq study of the transcriptome in three distinct areas of the neocortex when the tissue is sampled at different developmental stages. Whereas the pattern of results is complex and the findings elucidate the molecular mechanisms underlying the functional specification of different areas of the brain- and age-related changes, the main contribution of this article is in demonstrating the high potential of RNA-Seq for research on the specifics of gene expression among different cortical areas for normative (i.e., age-related) and aberrant (i.e., disorder-related) developmental trajectories.

The article by Hanna E. Stevens, Jessica Mariani, Gianfilippo Coppola, and Flora M. Vaccarino provides even more breathtaking possibilities of utilizing the molecular sciences in the quest for understanding the molecular machinery of typical and atypical behavioral development. This article is structured as a review of the field with illustrations of the concept. The authors provide an overview of the literature that has accumulated since the introduction of induced pluripotent stem cell (iPSC) technology. In principle, this technology made it possible to derive neuronal cells from somatic cells obtained from human individuals. In other words, it lifted the heavy cloud of doubt and softened the fierce debates around the usage of embryonic stem cells in scientific research, opening exciting new avenues for in vitro investigations and bridging the gap between molecular neuroscience and psychopathology and psychology. For the first time, this technology permits an examination of the cell biology, genetics, and epigenetics of neurons derived from particular

\section{References}

Caspi, A., Hariri, A. R., Holmes, A., Uher, R., \& Moffitt, T. E. (2010). Genetic sensitivity to the environment: The case of the serotonin transporter gene and its implications for studying complex diseases and traits. American Journal of Psychiatry, 167, 509-527.

Cicchetti, D. (1984). The emergence of developmental psychopathology. Child Development, 55, 1-7.

Cicchetti, D., \& Cohen, D. J. (1995). Perspectives on developmental psychopathology. In D. Cicchetti \& D. J. Cohen (Eds.), Developmental psychopathology: Vol. 1. Theory and method (pp. 3-20). New York: Wiley.

Collins, F. S. (2010). The language of life: DNA and the revolution in personalised medicine. New York: HarperCollins.

Falconer, D. S. (1965). The inheritance of liability to certain diseases, estimated from the incidence among relatives. Annals of Human Genetics, $29,51-76$.

Gottesman, I. I., \& Gould, T. D. (2003). The endophenotype concept in psychiatry: Etymology and strategic intentions. American Journal of Psychiatry, 160, 636-645. individuals (i.e., individuals with specific developmental disorders or individuals with well characterized behavioral profiles). The article delineates specific steps of the process: the selection and collection of specific cell types from an individual, recapitulating in vitro the developmental steps resulting in the rise of neuronal cells from stem cells, and the further differentiation of neuronal cells into cells specific to different brain structures, layers, and functional types. It also exemplifies the usage of the different modern genomic technologies for investigating the details of the transcription of human genes into messenger RNAs, the role of specific genetic variants in this transcription, and the mechanisms that further process these gene transcripts in the context of their control of developing cells. Although many (if not all) applications of iPSC technology pertain to studies of neurodevelopmental disorders such as autism spectrum disorders (Rett and Timothy syndromes), more possibilities for the utilization of iPSC open as the relevant science and technology develop. The authors conclude with the assertion that extensive knowledge of various aberrations in the development of the brain in general, particularly the cortex, will allow the field to better understand the tremendous amount of heterogeneity characteristic of virtually any developmental disorder. By grounding the heterogeneity of the specifics of early cellular mechanisms in such knowledge, we could ultimately boost our ability to diagnose and treat these developmental disorders.

We complete this introduction to the Special Issue with a statement that brings us back to the beginning. This Special Issue gives the field a comprehensive overview of its frontiers; it also provides a balanced view of the field's strengths and weaknesses, accomplishments and pitfalls. These articles present many interesting findings and ideas; they are also cognizant of many caveats. The summary is optimistic, but there is a lot of work to do. There is much room for exploration, inspiration, and excitement. Without a doubt, this work will be accomplished.

Gottesman, I., \& Shields, J. (1972). Schizophrenia and genetics: A twin study vantage point. New York: Academic Press.

Human Genome Program. (2008). Genomics and its impact on science and society: A 2008 primer. Washington, DC: US Department of Energy.

Mandelman, S. D., \& Grigorenko, E. L. (2012). BDNF Val66Met and cognition: All, none, or some? A meta-analysis of the genetic association. Genes, Brain and Behavior, 11, 127-136.

Plomin, R., \& Rutter, M. (1998). Child development, molecular genetics, and what to do with genes once they are found. Child Development, 69, 12231242.

Pritchard, J. K., \& Przeworski, M. (2001). Linkage disequilibrium in humans: Models and data. American Journal of Human Genetics, 69 , $1-14$

Rutter, M., \& Sroufe, L. A. (2000). Developmental psychopathology: Concepts and challenges. Development and Psychopathology, 12, 265-296.

Sroufe, L. A., \& Rutter, M. (1984). The domain of developmental psychopathology. Child Development, 55, 17-29. 